\title{
Combination Procedures to Provide Weight Loss: A Case Report
}

\section{Frederick Tiesenga, MD*, Shivani Sareen MS3, Karandeep Nijjar MS3, Harman Kainth MS3, Alyzeh Alibhai MS4}

General Surgery, West Suburban Medical Center, Chicago, USA.

*Corresponding Author: Frederick Tiesenga, MD, General Surgery, West Suburban Medical Center, Chicago, USA.

Received date: 13 October 2021; Accepted date: 18 October 2021; Published date: 20 October 2021

Citation: Tiesenga F, Sareen S, Nijjar K, Kainth H, Alibhai A (2021) Combination procedures to provide weight loss: A Case report. J Med Case Rep Case Series 2(17): https://doi.org/10.38207/JMCRCS/2021/0217181

Copyright: (C) 2021 Frederick Tiesenga, MD. This is an open-access article distributed under the terms of the Creative Commons Attribution License, which permits unrestricted use, distribution, and reproduction in any medium, provided the original author and source are credited.

\begin{abstract}
Obesity can complicate high blood pressure (hypertension), dyslipidemia, type 2 diabetes, coronary heart disease, stroke, and reduce life expectancy. Bariatric surgery is a standard method of producing consistent and sustainable weight loss. The laparoscopic adjustable gastric band is a reversible procedure that demonstrates restrictive weight loss. Typically, after improving diet, exercise habits, and the gastric band have failed to reach the patient's target weight, a vertical sleeve gastroscopy (VSG) is recommended. Vertical sleeve gastroscopy is often used once the laparoscopic band fails to achieve the patient's desired weight. VSG is an irreversible procedure that requires the removal of $80-85 \%$ of the stomach and then reattached to increase further satiety and weight loss. In addition to surgery, a low-calorie diet and modifiable lifestyle changes are adopted to sustain weight loss and prevent complications of obesity.
\end{abstract}

\section{Introduction}

Chronic obesity is a well-known problem that individuals face around the world. Weight loss is imperative to individuals whose risk factors can lead to diabetes, psychosocial disorders, and heart disease. Obesity is the second leading cause of preventable death after smoking in the United States. National institutes of health estimated 300,000 deaths per year are because of an obesity pandemic. Although an approach to primary prevention should be prioritized. Bariatric practitioners center on an effective therapy for rapid relief for those suffering from obesity. In association, pharmacotherapy has been an additive and synergistic approach alongside bariatric procedures that decrease hunger. If ideal conditions are not met physiologically, impairment of weight loss cannot be achieved.

\section{Case Presentation}

This case study presents a patient who has been dealing with obesity for over 30 years. The patient struggled to lose weight after having a vertical gastric sleeve procedure, over the span of 6 years the patient attempted to manage her own weight with diet and exercise. After many attempts, the patient elected to have a lap band around the existing sleeve. With a good diet and exercise, the patient lost some weight but was not satisfied with the results. With another 3-year lapse, the patient decided to have a SIPS procedure to help with further weight loss. After the $3^{\text {rd }}$ procedure, the patient was happy with the results and continued to lose weight and achieve her goals.

\section{Discussion}

Although a gastric bypass would have been the more common way to introduce more weight loss in this patient, the surgeon could place a laparoscopic adjustable gastric band successfully. The patient reported that she could not eat as much as she did before the initiation of resistance. Both the patient and the doctor noticed a significant yet steady weight loss at that stage. The patient was alleviated, preventing risk factors that would decrease the patient's life expectancy and improved the lifestyle that the patient ultimately desired.

\section{Conclusion}

Surgical intervention is critical for those experiencing obesity and the risks of disease. The long-term effects of surgical weight loss have been considered, so they meet the standards of rapid, sustainable reduction in weight. Bariatric surgery is the primary foundation of life longevity in modern practice. There is sufficient evidence suggesting Laparoscopic adjustable band and Vertical sleeve gastrectomy are reliable procedures that benefit bariatric patients. It remains to be seen if the patients receiving the surgical procedures are effective therapy to overcome treatment resistance weight loss. A Laparoscopic adjustable band after a vertical sleeve gastrectomy is a rare recommendation, providing the risk factors, but can be improved over time to combat morbid obesity.

\section{Case Presentation}

A 58-year-old female with a past medical-surgical history of gastric sleeve, 2012, and laparoscopic adjustable gastric band, 2018, comes into the outpatient clinic to request a stomach intestinal pylorus sparing surgery in 2021. The patient had been struggling with chronic obesity for the past 30 years of her life. After the patient started to develop comorbid conditions that commonly occur with obesity, such as borderline diabetes, hypertension, and shortness of breath after walking a few blocks, the patient came to the outpatient surgery clinic 
for consultation of a gastric sleeve in 2011. The patient was determined to be a good candidate for gastric sleeve surgery, and the surgery was conducted in 2012. Three months after the surgery, the patient presented to the outpatient surgery clinic with concerns about not losing weight for the past four weeks. She also wanted to be evaluated because she could eat large meals, and she did not feel full even after the surgery. The patient was counseled on the 800 -calorie diet and an exercise regimen to help with weight loss. In 2017, the patient presented to the outpatient surgery clinic to consult a laparoscopic adjustable gastric band. The patient dictated symptoms of experiencing heartburn and acid reflux for many months. After careful evaluation of her symptoms, the patient was given a

\section{Discussion}

A Vertical Sleeve Gastroscopy (VSG) is a current procedure commonly used for obese patients with help for the reduction of excessive adipose tissue. This procedure consists of a reduction in the size of the stomach by cutting out $70-80 \%$ of the stomach. The remaining portion of the stomach is then reattached and made into a banana shape pouch. This procedure is permanent, and it is irreversible. With the VSG, a patient can lose 50-70\% of body fat over 18 months. With any gastric procedure, the patients must alter their lifestyle and eating habits. More commonly, patients are put on a low-calorie diet to sustain weight loss. Alongside the management of weight, it is crucial to supplement patients with a multivitamin because patients will have a decreased absorption of all fat-soluble vitamins. Gastric procedures not only help with the reduction of adipose tissue, but they also help with other comorbidities and, in some cases, can help treat other conditions a patient may be diagnosed with.

A Laparoscopic Adjustable Gastric Band (LAGB) is more colloquially known as a Lap-Band. This procedure is also a standard approach for reducing excessive adipose tissue. This procedure will entail a small donut-liked device placed around the upper portion of

\section{Conclusion}

After struggling with chronic obesity for the past 30 years of her life, the patient started to develop comorbid conditions that commonly occur with obesity, such as borderline diabetes, hypertension, and shortness of breath; after walking a few blocks, the patient came to the outpatient surgery clinic for consultation of a gastric sleeve. She had a gastric sleeve procedure that was done in 2012. After the procedure, the patient was having trouble losing weight; over four weeks, the patient had not lost any sustainable amount of weight. After attempting weight loss with the gastric sleeve over six years, the laparoscopic band in 2018. In 2021, the patient presents to the clinic to request a small intestinal pylorus sparing surgery (SIPS). The patient felt she could not reach her goals without additional surgical intervention. The patient was also shown diet and lifestyle modifications, including increasing daily water intake and protein. The patient was also encouraged to exercise for 15 to 30 minutes daily. The patient returned to the clinic again for not losing weight and her BMI still being well over 40. After careful evaluation of her symptoms, the small intestinal pylorus-sparing surgery was conducted in 2021. After the small intestinal pylorus-sparing procedure, the patient experienced gradual weight loss.

the stomach, which will reduce the size of usable space in the stomach down to $20 \%$ of the previous size. The procedure will allow the patient to eat smaller portions and reach early satiety, which will help the patient with portion control. The adjustment of the band will allow patients control on constriction making it more customizable to their lifestyle. Alongside the adjustment of the band, the patient will also be placed on a controlled diet and multivitamin plan to help sustain their weight loss and supplement all the fat-soluble vitamins.

A stomach intestinal pylorus sparing surgery (SIPS) is a procedure that has the benefits of both the gastric sleeve and the gastric bypass surgery. The SIPS combines both procedures and allows optimal weight loss, allowing patients to lose up to 80-90\% percent of their excess adipose tissue over 18-20 months. The SIPS procedure has two parts; the first part is the gastric sleeve, removing $70-80 \%$ of the stomach and forming a banana shape pouch. The second part of the SIPS procedure involves dividing the top part of the small bowel to the top of the small intestine. As a result, food bypasses the part of the small intestine. This leads to a reduction in consumption and absorption of calories, which allows for optimal weight loss

patient was not satisfied with her results; she received an adjustable band in 2018. After the procedure, the patient followed up with the outpatient clinic and made several adjustments over three years. In 2021 the patient who had plateaued with her weight loss journey had consulted a physician to receive a SIPS procedure. Subsequent consultation with a physician, the patient was instructed to followthe diet plan and continue with her fat-soluble vitamins. Following the procedure, the patient lost weight at a sustainable level and is on track to achieve her personal goals. 
Ethical Approval: Obtained

\section{Source of Funding: None}

Research Registration Unique Identify Numb (UIN):

None

Guarantors: H.G., K.N., S.S., H.K

\section{References}

1. Slater GH, Ren CJ, Siegel N, Williams T, Barr D, Wolfe B, Fielding GA (2004) Serum fat-soluble vitamin deficiency and abnormal calcium metabolism after malabsorptive bariatric surgery. Journal of Gastrointestinal Surgery: Official Journal of the Society for Surgery of the Alimentary Tract, 8(1): 48-55.

2. Amy Banks MD, R Harrell MD, J Foote MD, BAND OVER SLEEVE: A SAFE ALTERNATIVE TO ACHIEVE FURTHER
Author Contribution: K.N., H.G writing, draft preparation, investigator. H.K., S.S Writing, draft preparation, supervision. S.S., K.N. writing, draft preparation, editor, reviewer

Conflict of Interest Disclosure: The authors declare that they have no financial conflict of interest regarding the content of this report.

\section{WEIGHT LOSS AND REDUCTION OF CO-MORBIDITIES}

Grand Rapids Medical Education Partners, Michigan State University and Grand Health Partners.

3. Dixon JB, Straznicky NE, Lambert EA, Schlaich MP, Lambert GW (2012) Laparoscopic Adjustable Gastric Banding and Other Devices for the Management of Obesity, AHA JOURNALS. 126(6): 774-785. 